\title{
Expression of PAX8 in normal and neoplastic renal tissues: an immunohistochemical study
}

\author{
Guo-Xia Tong, Woojin M Yu, Nike T Beaubier, Erin M Weeden, Diane Hamele-Bena, \\ Mahesh M Mansukhani and Kathleen M O’Toole
}

Department of Pathology, Columbia University College of Physicians and Surgeons, New York, NY, USA

\begin{abstract}
Cell-lineage-specific transcription factors are a group of regulatory proteins expressed in embryonic, differentiated, or neoplastic cells of the same lineage and represent a valuable repertoire of tissue-specific markers for the diagnosis of human tumors. Together with PAX2, PAX8 is a nephric-lineage transcription factor and is required for the establishment of renal-lineage cells and the formation of the kidney. In contrast to PAX2, little is known about the expression of PAX8 in adult kidney and renal tumors. In this study, we used immunohistochemistry to investigate the expression of $P A X 8$ in adult human kidney and renal epithelial tumors. We report here that $P A X 8$ was detected in renal epithelial cells in all segments of renal tubules from the proximal tubules to the renal papillae and in the parietal cells of Bowman's capsule in the adult kidney. PAX8 was also present in $98 \%$ of clear cell renal cell carcinomas (RCCs), $90 \%$ of papillary RCCs, and $95 \%$ of oncocytomas, similar to PAX2. In addition, PAX8 was found in $82 \%$ of chromophobe RCCs, $71 \%$ of sarcomatoid components of RCCs, and $100 \%(2 / 2)$ of renal medullary carcinomas. Overall, PAX8 was detected in $85 \%$ of metastatic renal tumors. Interestingly, expression of PAX8 was noted in some urothelial cells in the renal pelvis and ureters and $\sim 23 \%$ of urothelial carcinomas of the renal pelvis, but not in the urothelium or urothelial carcinomas of the urinary bladder; this probably underlines the different embryonic origins of urothelial cells in the upper and lower urinary tracts. As shown in this study, PAX8 is widely expressed in normal and neoplastic renal tissues. $P A X 8$ may be a useful additional marker for renal epithelial tumors; however, its specificity and sensitivity await further investigation.
\end{abstract}

Modern Pathology (2009) 22, 1218-1227; doi:10.1038/modpathol.2009.88; published online 12 June 2009

Keywords: $P A X 8$; renal epithelial tumors; urothelial carcinoma; immunohistochemistry

Cell-lineage-specific transcription factors have recently emerged as valuable diagnostic markers for human tumors. These proteins are expressed in limited types of embryonic tissues and are required for the growth and differentiation of these tissues into highly specialized tissues or organs. As some proteins continue to be expressed in the differentiated tissues during adult life or in the neoplastic tissues of the same cell lineage, detection of these proteins could indicate the primary site or the differentiation trait of a tumor. The well-established diagnostic markers in this category include TTF-1, ${ }^{1,2}$ CDX $2,{ }^{3} \mathrm{MITF}^{4}$ and myogenin. ${ }^{5,6}$

Renal cell carcinoma (RCC) is known for its predilection for vascular invasion and distant

Correspondence: Dr G-X Tong, MD, PhD, Department of Pathology, Columbia University College of Physicians and Surgeons, 630 West 168th Street, Room VC14-238, New York, NY 10032, USA. E-mail: gt2125@columbia.edu

Received 02 March 2009; revised and accepted 04 May 2009; published online 12 June 2009 metastases. A significant number of cases present with extra-renal growth or metastasis at the time of diagnosis or after surgery. ${ }^{7}$ On account of its diverse histologic features, metastatic RCC can be a diagnostic challenge and frequently requires ancillary tests, such as immunohistochemistry, for confirmation. ${ }^{8}$ Many makers including cytokeratins (CK), CK7, CK20, EMA, vimentin, ${ }^{9}$ c-kit, ${ }^{10}$ P504S, ${ }^{11}$ RCCmarker, ${ }^{12,13}$ aquaporin- $1,{ }^{14}$ CD10, ${ }^{15}$ and carbonic anhydrase 9 (CAIX) $^{16,17}$ have been used in various panels with variable sensitivity and specificity.

$P A X 2$ and $P A X 8$, members of PAX gene family, are nephric cell-lineage transcription factors and have important functions in the renal organogenesis. ${ }^{18,19}$ Both are expressed in Wolffian ducts, the precursor tissues for the pronephros and the ureteric bud. ${ }^{20,21}$ Either $P A X 8$ or $P A X 2$ alone is sufficient for the formation of the pronephros and they are, therefore, likely functionally redundant. ${ }^{21} P A X 2$ seems to be more important than $P A X 8$ for the development of the mesonephros (second kidney) and metanephros (third and final kidney). Nevertheless, $P A X 8$ is 
coexpressed with PAX2 in almost every stage of kidney development, such as in Wolffian ducts, ureteric buds, and the transformed mesenchymal tissues, during the formation of metanephros. ${ }^{20,21}$ The diagnostic applications of PAX2 have been studied more extensively than PAX8. It has been detected in Wilms tumors, ${ }^{22}$ RCCs, ${ }^{23,24}$ and nephrogenic adenomas ${ }^{25,26}$ and has been used as a diagnostic marker for benign and malignant lesions of renal origin. ${ }^{27}$ PAX8 was detected in pediatric Wilms tumors ${ }^{28}$ and recently in nephrogenic adenomas ${ }^{29}$ however, there is little information available about its expression in adult normal or neoplastic renal tissues. Here, we report our findings of $P A X 8$ expression in human adult kidney and renal epithelial tumors using immunohistochemistry.

\section{Material and methods}

One hundred and forty-three primary and 39 metastatic renal epithelial tumors were used in this study. The primary renal epithelial tumor samples were from radical nephrectomy specimens and were constructed into five-tissue microarrays with three representative $1 \mathrm{~mm}$ cores from each case. Conventional tissue sections from the metastatic tumors were used. Also included were 40 urothelial carcinomas of the urinary bladder in one-tissue microarray, which was created in the same fashion as for the primary renal epithelial tumors. Normal tissue controls included 40 cores of uninvolved renal parenchyma contained in the tissue microarrays as well as tissue sections from the uninvolved renal pelvic areas $(N=5)$, ureters $(N=5)$, and urinary bladder $(N=10)$.

$P A X 8$ immunohistochemical staining was performed in a Dako autostainer with rabbit anti-PAX8 polyclonal antibody (1:100) (Poteintech, Inc, Chicago, IL, USA). In brief, the paraffin-embedded tissue sections ( $5 \mu \mathrm{m}$ thick) were obtained from the TMA or tissue blocks and baked overnight in the oven at $60^{\circ} \mathrm{C}$. The sections were de-paraffinized and dehydrated sequentially with PRO-PAR clearant (Anatech, Battle Creek, MI, USA) in a series of descending concentrations of alcohol and water. Antigen retrieval was done by steaming the sections for $40 \mathrm{~min}$ and cooling for $20 \mathrm{~min}$ in Cell marque in Trilogy with ethylenediaminetetraacetic acid (EDTA) solution. After antigen retrieval, the sections were incubated with Tris-buffered solution with Tween-20 for $5 \mathrm{~min}$ and then loaded to the Dakoautomated-staining system. The EnVision system (Dako) was used to visualize the primary antibody with hematoxylin counterstaining.

The sensitivity and specificity of $P A X 8$ antibody were validated with an array of normal tissues, and only distinct nuclear staining was considered positive for $P A X 8 .^{29} \mathrm{~A}$ diffuse positive staining is referred to as $>50 \%$ of tumor cells staining positive for $P A X 8$; otherwise, staining was considered focally positive. For metastatic RCCs, cases with $>5 \%$ of tumor cells staining for $P A X 8$ were considered to be positive. The intensity of $P A X 8$ staining in tumors was graded as weak $(1+)$, moderate $(2+)$, and strong $(3+)$.

\section{Results}

Expression of $P A X 8$ in Normal Adult Kidney, Ureters, and the Urinary Bladder

$P A X 8$ staining was detected in the epithelial cells of the proximal and distal renal tubules, loops of Henle, collecting ducts, and the parietal epithelial cells of Bowman's capsule (Figure 1a and b). The staining was always stronger in the distal tubules, parietal cells of Bowman's capsule, and collecting ducts $(3+)$ than in the proximal tubules $(1+)$, in which nonspecific cytoplasmic staining was also observed. Strong $P A X 8$ staining $(3+)$ was detected in all layers of the lining urothelial cells in the renal papilla and in the initial portion of the renal pelvis. In the distal portion of the renal pelvis and ureters, $P A X 8$ positive cells were gradually limited to the intermediate or basal layers of the urothelium with decreased staining intensity $(2+)$ (Figure 1c-e). There was no $P A X 8$ staining in the urothelium of the urinary bladder (Figure 1f). PAX8 staining was neither detected in the podocytes, mesangial cells, endothelial cells, nor in the interstitium or lymphvascular structures of the kidney.

\section{Expression of $P A X 8$ in Renal Epithelial Tumors}

The results of $P A X 8$ staining in primary renal epithelial tumors are summarized in Table 1 . The cohort of primary renal tumors included 66 clear cell RCCs, which were from 41 men and 25 women with ages ranging from 37 to 86 years and a mean age of 64 . The tumor sizes varied from 1.5 to $22 \mathrm{~cm}$ (mean size $=7.4 \mathrm{~cm}$ ). These included 44 purely clear cell RCCs, 15 mixed clear cell and granular cell pattern cases, and 7 clear cell RCCs with sarcomatous differentiation. By the Fuhrman grading system, 3 cases had tumors with nuclear grade 1, 25 cases with nuclear grade 2, 27 cases with nuclear grade 3, and 11 with cases with nuclear grade 4 . Of these cases, tumors were at stage I in 15 patients, stage II in 24 patients, stage IIIA in 14 patients, and stage IV in 13 patients. PAX8 was detected in 58 of $59(98 \%)$ clear cell RCCs (including the 15 cases with mixed clear and granular cell types), with diffuse positive staining in 52 cases (Figure 2a and $b$ ) and focal positive staining in 6 cases. For those cases with diffusely positive $P A X 8$ staining, the staining intensity was variable with weak staining $(1+)$ in 10 cases, moderate staining $(2+)$ in 25 cases, and strong staining $(3+)$ in 17 cases. The focally positive cases usually had weak staining as well, with one exception in which focal strong 

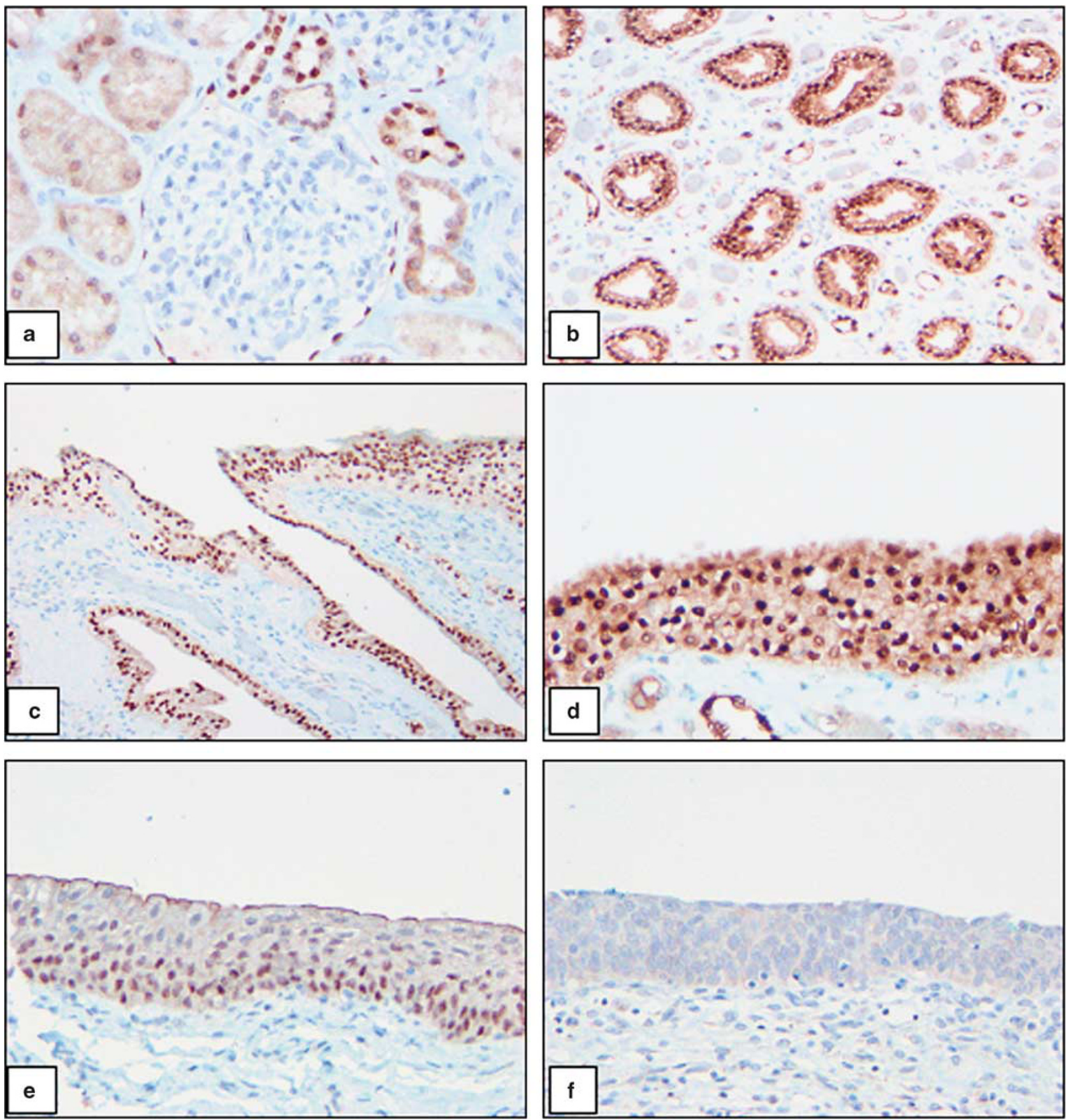

Figure 1 Immunohistochemical staining of $P A X 8$ in normal adult kidney. Immunoreactive $P A X 8$ was detected in proximal tubules and the parietal cells of the Bowmen's capsule $(\mathbf{a}, 200 \times)$, in distal renal tubule cells and loop of Henle $(\mathbf{b}, 200 \times)$, in collecting duct and renal papillae $(\mathbf{c}, 200 \times)$, the urothelium of renal pelvis $(\mathbf{d}, 200 \times)$, the urothelium of the ureters $(\mathbf{e}, 200 \times)$, but not in the urothelium of the urinary bladder $(\mathbf{f}, 200 \times)$.

staining was noted. The only negative case was a conventional clear cell RCC, $9 \mathrm{~cm}$ in size, with nuclear grade 3 and stage II disease from a 71-yearold man. Weak $(1+)$ or moderated $(2+)$ staining of $P A X 8$ was detected in the malignant spindle cells or rhabdoid tumor cells in five of seven (71\%) RCCs with sarcomatous components, diffusely in three and focally in two cases (Figure 2c and d). There seemed to be no correlation between the staining intensity and nuclear grades or tumor stages.

Twenty-one primary papillary RCCs were included in this study (18 males, 3 females, age range from 44 to 85 years, mean age 70 years) and the tumor size varied from 2.2 to $11.7 \mathrm{~cm}$ (average size $5.9 \mathrm{~cm}$ ). Seventeen cases were type 1 papillary RCCs at either stage I or II, and nine or eight patients, 
Table 1 Summary of PAX8 immunostaining in primary renal epithelial tumors

\begin{tabular}{|c|c|c|c|c|c|c|}
\hline & Number of cases & Negative stain & Focally stain & Diffuse 1+ & Diffuse 2+ & Diffuse 3+ \\
\hline RCC, clear cell & 59 & 1 & 6 & 10 & 28 & 18 \\
\hline RCC, sarcomatous component & 7 & 2 & 3 & 1 & 1 & 0 \\
\hline RCC, papillary & 21 & 2 & 0 & 1 & 3 & 15 \\
\hline RCC, chromophobe & 11 & 2 & 0 & 4 & 4 & 1 \\
\hline Renal medullary carcinoma & 2 & 0 & 0 & 0 & 2 & 0 \\
\hline Oncocytoma & 22 & 1 & 0 & 0 & 6 & 15 \\
\hline UC, renal pelvis & 17 & 13 & 0 & 0 & 4 & 0 \\
\hline UC, ureter & 2 & 2 & 0 & 0 & 0 & 0 \\
\hline UC, urinary bladder & 40 & 40 & 0 & 0 & 0 & 0 \\
\hline
\end{tabular}

RCC, renal cell carcinoma; UC, urothelial carcinoma.

respectively. Four cases were type 2 papillary RCCs that were either stage II or III. PAX 8 was detected in 19/21 (90\%) of the papillary RCCs, including 16 type 1 and 3 type 2 tumors. The $P A X 8$ staining was diffuse and strong $(3+)$ in 15 cases (Figure 2e and f) (14 type 1 and 1 type 2 tumors) and moderate $(2+)$ in 4 cases (2 type 1 and 2 type 2 tumors). The two $P A X 8$ negative cases included one of the type 1 and one of the type 2 tumors.

There were 11 chromophobe RCCs in this study (6 male, 5 female, age range from 28 to 75 years, mean age 58 years) and the tumor sizes varied from 1 to $14 \mathrm{~cm}$ (mean tumor size $4.9 \mathrm{~cm}$ ). The tumors were stage I in six patients, stage II in four patients, and stage III with lymph node metastases in one patient. Nine of $11(82 \%)$ chromophobe RCCs were diffusely positive for $P A X 8$ (Figure 3a and b), with moderate $(2+)$ in four and weak intensity $(1+)$ in five cases.

There were two renal medullary carcinomas in this study; one was a 31-year-old African American man with sickle cell trait and the other was a 34year-old woman without a known history of sickle cell trait/anemia. The tumors were 10 and $5 \mathrm{~cm}$, respectively, and located mainly in the renal medulla with extensive involvement of the renal cortices, renal pelvis, ureters-pelvic junctions, and hilar soft tissue. Histologically, both tumors were composed of poorly differentiated neoplastic cells in sheets, cords, or nests, with highgrade nuclear features, brisk mitotic activity, and necrosis. Both tumors were diffusively positive for $P A X 8$ with moderate-staining intensity $(2+)$ (Figure $3 e$ and $f$ ).

The study of primary renal tumors also included 22 oncocytomas (16 male, 6 female, age range from 43 to 80 years, mean age 68 years) and the tumors varied from 1.5 to $10.7 \mathrm{~cm}$ (mean size $4.8 \mathrm{~cm}$ ). Twenty-one of 22 (95\%) oncocytomas were diffusely positive for $P A X 8$ with strong staining $(3+)$ (Figure 3c and d) in 15 cases and moderate staining $(2+)$ in 6 cases. The only oncocytoma negative for $P A X 8$ was an 8-cm tumor from a 63-year-old man with unremarkable histologic features.

\section{Expression of $P A X 8$ in Urothelial Carcinoma of the Renal Pelvis, Ureters, and Urinary Bladder}

The expression of $P A X 8$ was also studied in urothelial carcinomas of the upper and lower urinary tract because of the discrepancy of its expression in the normal urothelial cells of these areas. The cases included 17, 2, and 40 urothelial carcinomas of the renal pelvis, the ureters, and the urinary bladder, respectively. The nuclear features were graded by a three-tier system. The nuclei of 10 , 6 , and 1 urothelial carcinomas of the renal pelvis were graded as 3,2 , and 1 , respectively. The two urothelial carcinomas of the ureter were nuclear grade 3. Twenty-seven of the 40 urothelial carcinomas of the bladder were graded as nuclear grade 3 and the rest were nuclear grade 2. Diffuse and moderately strong staining $(2+)$ of $P A X 8$ was detected in $4(\sim 23 \%)$ of the 17 urothelial carcinomas of the renal pelvis (Figure 4), including three cases with nuclear grade 2 and one case with nuclear grade 3 . There was no $P A X 8$ detected in the 2 urothelial carcinoma of the ureters or the 40 urothelial carcinomas of the urinary bladder. The results are also summarized in Table 1.

\section{Expression of $P A X 8$ in Metastatic RCCs}

Of the 39 metastatic RCCs, 5 were in regional lymph nodes, 6 in lung, 4 in liver, 7 in bone or soft tissue, 5 in brain, 5 in adrenal glands, 6 in pancreas, and 1 in thyroid. Of these, 32 were clear cell type, 3 papillary, 2 poorly differentiated, 1 displayed rhabdoid features, and 1 had a spindle cell component. $P A X 8$ was detected in $33(85 \%)$ of 39 metastatic RCCs, with weak staining $(1+)$ in 11 , moderate staining $(2+)$ in 17 , and strong straining $(3+)$ in 5 cases. Five of the clear cell types and the tumor with rhabdoid features were negative for $P A X 8$. The spindle cell RCC was weakly positive for $P A X 8$, whereas the two poorly differentiated metastatic RCCs were moderately positive. The results are summarized in Table 2. There was no PAX8 staining in the parenchyma of the lung, liver, 

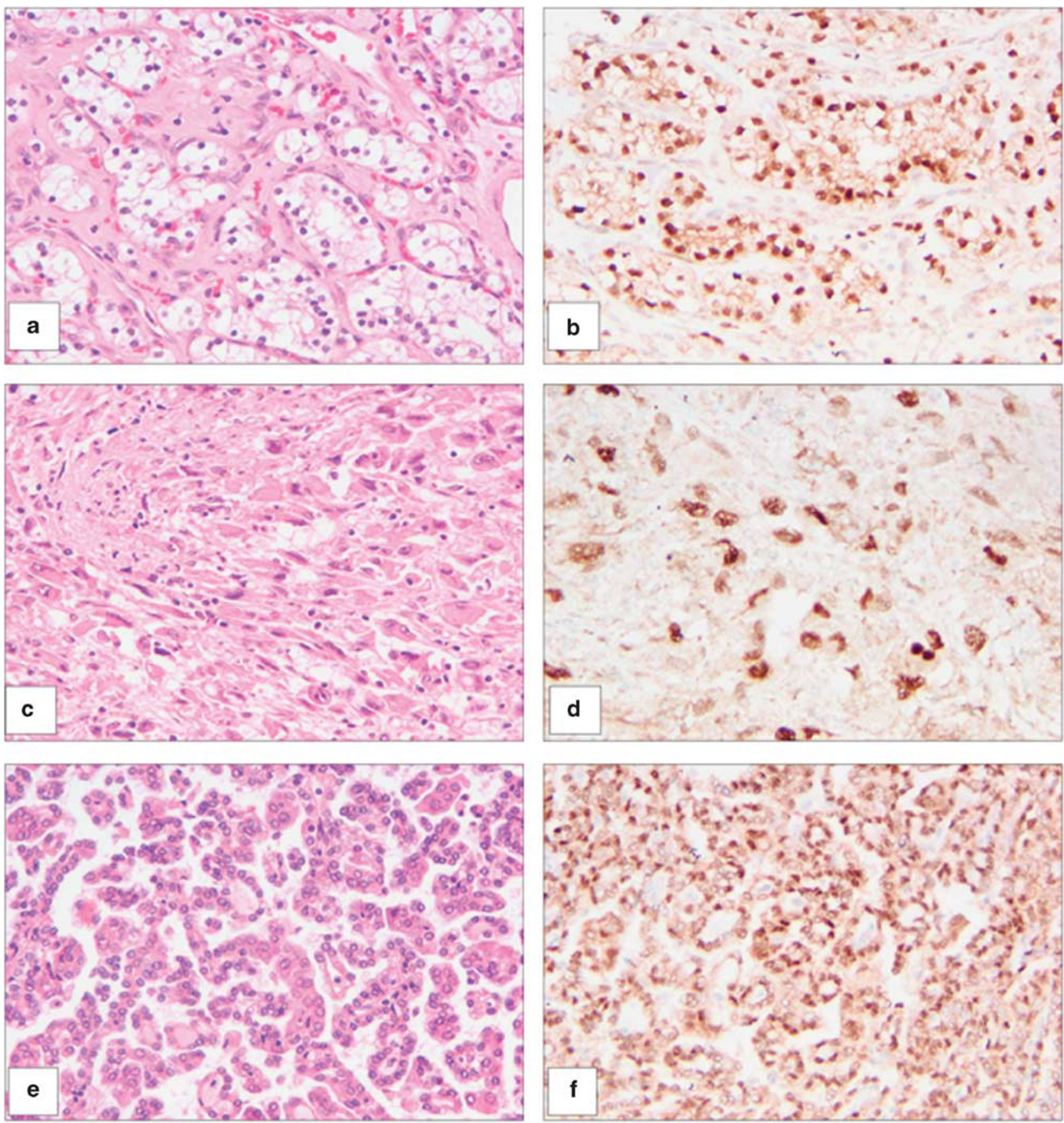

Figure 2 Examples of immunohistochemical staining of $P A X 8$ in renal epithelial tumors. Immunoreactive $P A X 8$ was detected in clear cell RCCs (a, H\&E; b, PAX8; $200 \times)$, sarcomtoid component (rhbadoid) of RCCs (c, H\&E; d, PAX8; $200 \times)$, and papillary RCCs (e, H\&E; f, $P A X 8 ; 200 \times)$.

adrenal tissues, or pancreas. Some lymphocytes did stain positive for PAX8; however, they are morphologically distinct from the metastatic carcinoma and will not cause diagnostic difficulties.

\section{Discussion}

$P A X 8$ and $P A X 2$ are important developmental transcription factors in renal organogenesis. ${ }^{18,19,30}$
Expression of $P A X 8$ in the anlagen tissues for the future kidney occurs slightly earlier than $P A X 2$. Thereafter, both $P A X 8$ and $P A X 2$ have similar expression patterns in the course of kidney formation. Either $P A X 8$ or $P A X 2$ is necessary and sufficient for the establishment of nephric-lineage cells and the formation of first embryonic kidneys, the pronephros. ${ }^{30}$ After the formation of the pronephros, PAX2 seemingly has more important functions in the continuation of kidney development 

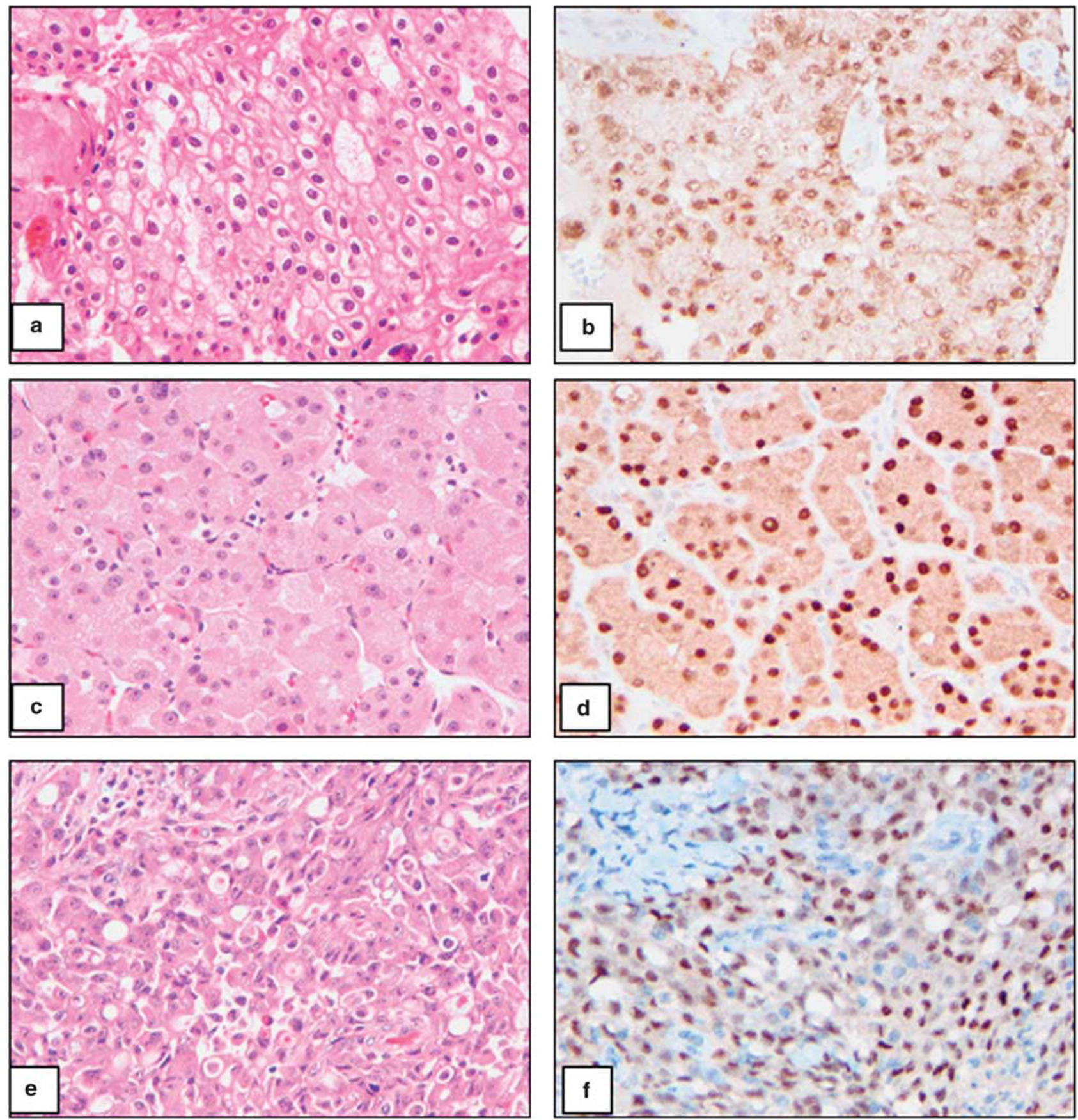

Figure 3 Examples of immunohistochemical staining of $P A X 8$ in renal epithelial tumors. Immunoreactive $P A X 8$ was detected in chromophobe RCCs (a, H\&E; b, PAX8; $200 \times)$ ), in oncocytomas (c, H\&E; d, PAX8; $200 \times$ ), and in renal medullary carcinomas (e, H\&E; f, PAX8; $200 \times)$.

and eventual formation of metanephros. Absence or malformation of kidney is associated with $P A X 2$ mutation alone, whereas mice with PAX8 deletions have normal kidneys. ${ }^{18,19}$ However, the additional mutation of $P A X 8$ would exacerbate the kidney defects caused by PAX2 mutation. ${ }^{21}$ It has been reported that $P A X 8$ expression is decreased or absent in mature mice kidneys; however, its expression and cellular distribution in adult human kidneys are largely unknown. ${ }^{28}$ We showed here by immunohistochemistry that $P A X 8$ is expressed in adult human kidney epithelial cells of the proximal and distal convoluted tubules, loops of Henle, collecting ducts, and the parietal cells of Bowman's capsule, with lower levels of expression in the proximal renal tubules than in other segments. $P A X 8$ expression was not detected in mesangial cells, endothelial cells, and visceral cells of Bowman's capsules in the glomeruli. No $P A X 8$ staining was detected in the stroma or renal vasculature. The 

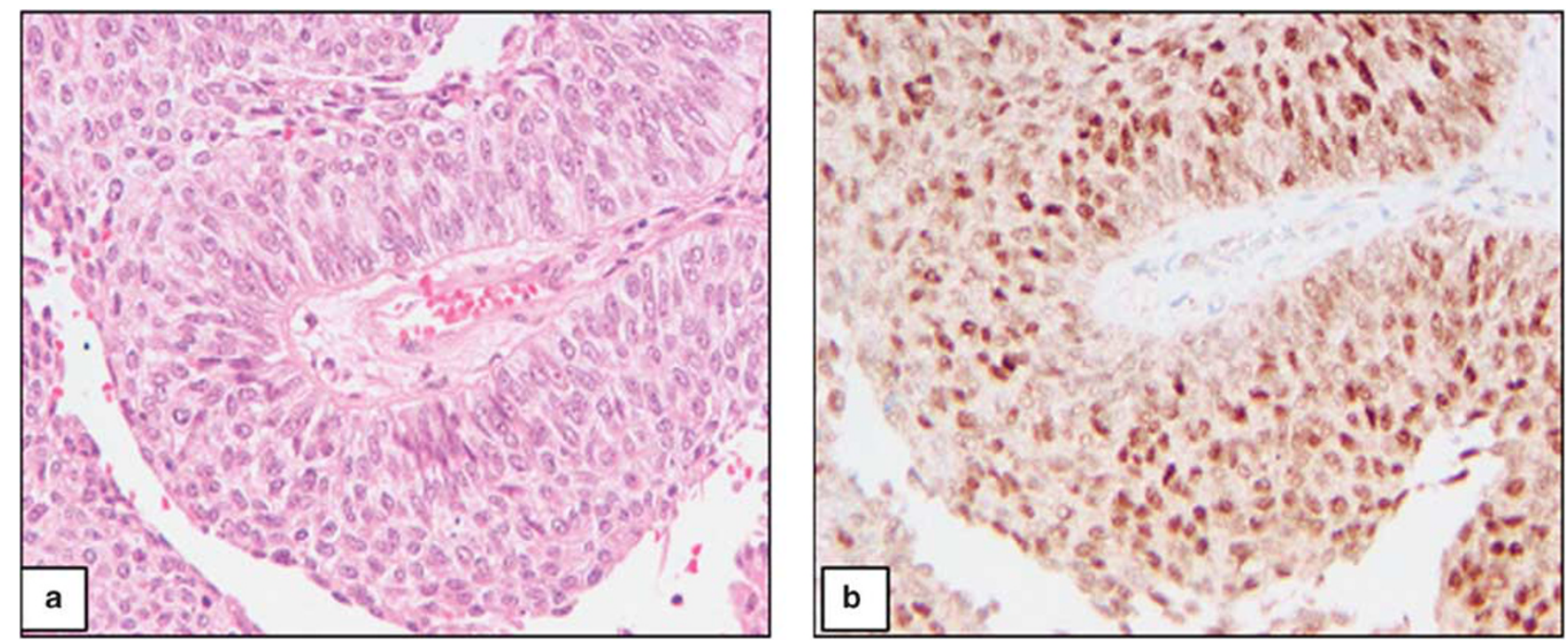

Figure 4 Examples of immunohistochemical staining of $P A X 8$ in urothelial carcinomas of the renal pelvis $(\mathbf{a}, \mathrm{H} \& \mathrm{E} ; \mathbf{b}, P A X 8 ; 200 \times)$.

Table 2 Summary of PAX8 staining in metastatic renal cell carcinomas

\begin{tabular}{|c|c|c|c|c|c|}
\hline Types & Number of cases & Negative staining & Weak (1+) & Moderate (2+) & Strong $(3+)$ \\
\hline RCC, clear cell & 32 & 5 & 10 & 13 & 4 \\
\hline RCC, papillary & 3 & 0 & 0 & 2 & 1 \\
\hline RCC, poorly differentiated & 2 & 0 & 0 & 2 & 0 \\
\hline RCC, sarcomatous features & 2 & 1 & 1 & 0 & 0 \\
\hline
\end{tabular}

RCC, renal cell carcinoma.

functions of $P A X 8$ in adult kidneys have not been elucidated, but it is probably involved in maintaining the differentiated features and specialized functions of renal epithelial cells. ${ }^{21,31}$

Renal epithelial cells differ in their embryonic origins. $^{32}$ The ureteric bud gives rise to the collecting duct system by dichotomous branching. The principal cells of the collecting duct are epithelial in origin and are the direct progenies of the epithelial cells of the ureteric bud. ${ }^{33}$ However, the tubular cells proximal to the loop of Henle (the so-called 'proximal nephron') are derived from the mesenchymal cells surrounding the branching tips of the ureteric bud. The mesenchymal cells are transformed into epithelial cells and form the proximal portion of the nephron from the proximal tubules to the loops of Henle. The mesenchymal-epithelial transformation is mediated by mutual induction between the mesenchymal cells and the branching ureteric bud. ${ }^{33}$ The differences in embryonic origins and functions in the different segments of nephron may have contributed to the diversity of morphologic patterns, molecular, and immunohistochemical phenotypes of common renal epithelial tumors. ${ }^{34}$ On account of these variable features and the absence of renal-specific positive markers, ${ }^{13}$ a panel of immunohistochemical markers is always required to substantiate the diagnosis and classification of renal epithelial tumors. Therefore, renal-specific immunohistochemical markers are of great diagnostic interest.

Renal epithelial tumors, especially clear cell RCCs, are typical for the coexpression of both epithelial and mesenchymal differentiation markers, CK, and vimentin, which are always included in the differential immunohistochemistry panels. Coexpression of CK and vimentin may be a reflection of mesenchymal origin of the proximal renal epithelial cells from which most common renal epithelial tumors are purportedly derived..$^{35}$ Additional markers, such as CK7, CK20, EMA, CD10, c-kit, and CAIX, have been used for the diagnosis and classification of renal epithelial tumors. However, the specificity and sensitivity of these markers are limited because the expression of these markers is not limited to the renal tissues and there is significant overlapping with tumors of other tissue origins. ${ }^{9-11,15-17}$ RCC-marker ${ }^{12,13}$ and aquaporin- ${ }^{14}$ are the markers with relative high specificity to the renal tissue, which have improved the diagnostic specificity and sensitivity of renal epithelial tumors by immunohistochemistry. However, these markers have some intrinsic limitations. As they are usually the terminal differentiation products of renal epithelial cells, they appear in the late stages of development and on the cell surface of the fully 
differentiated cells, and they are usually less detectable in poorly differentiated malignant tumors. In addition, most of these proteins were later found not to be as specific as initially claimed. ${ }^{12-14}$ In contrast, the cell-lineage-specific transcription factors are expressed much earlier during the development and most of them are the 'initiators' of specific cellular differentiation. They appear before the terminal differentiation proteins and are more frequently detected in poorly differentiated tumors. Expression of these transcription factors is usually limited to normal and neoplastic tissues of the same cellular lineages. In addition, these proteins have distinct nuclear staining, which makes the interpretation of immunohistochemistry staining more reliable and more appealing as a resource for the identification of tissue-specific immunohistochemistry markers. The specificities have been upheld for most transcription factors in use. ${ }^{1-6} P A X 2$ is the first renal-specific transcription factor whose diagnostic application in renal epithelial tumors has been investigated extensively. ${ }^{22-24,27}$ The distribution pattern of $P A X 8$ showed in this study indicates that it may be used as a pan-renalepithelial marker, in addition to its functional counterpart $P A X 2$.

PAX2 was shown in $84-93 \%$ of clear cell RCCs, $85-100 \%$ of papillary RCCs, and $87 \%$ of oncocytomas. ${ }^{23,24,36}$ We show here that $98 \%$ of clear cell RCCs, $90 \%$ of papillary RCCs, and 95\% of oncocytomas were positive for $P A X 8$, frequencies which are similar or better than for PAX2. PAX8 was also detected in $85 \%$ of metastatic RCCs in this study. In addition, $P A X 8$ was detected in $82 \%$ of chromophobe RCCs, $100 \%$ of renal medullary carcinomas, and $71 \%$ of sarcomatoid component of RCC, which are usually negative for $P A X 2$, aquaporin-1, and CAIX. This suggests that $P A X 8$ has a broader diagnostic application than these markers. For example, detection of $P A X 8$ in a poorly differentiated carcinoma of the kidney would help to establish the diagnosis of rare and high-grade primary renal tumor, such as renal medullary or collecting duct carcinomas.

We also observed a differential expression pattern of $P A X 8$ in the urothelium of upper and lower urinary tract. $P A X 8$ was detected in the full thickness of the urothelium in the renal papillae. $P A X 8$ positive cells decreased in number in the renal pelvis, ureters, and were gradually confined to the intermediate and basal layers. No $P A X 8$ was detected in the urothelium of the urinary bladder or urethra. This expression pattern may reflect the different embryonic origins of the urothelium of the upper and lower urinary tracts. As for the collecting ducts, the renal pelvis and ureters are also derived from the ureteric bud. ${ }^{32}$ When the proximal portion of the ureteric buds gives rise to the collecting duct system by dichotomous branching, its distal portion distends and elongates to form the renal pelvis. While extending distally, the ureteric bud acquires smooth muscle and connective tissues to form the tubular ureters. At the same time, the epithelial cells of the ureteric bud undergo morphologic and functional transformation from a single layer of cuboidal cells to the urothelium consisting of multiple layers of cells at different differentiation stages to cover the inner surface of the renal pelvis and ureters. Therefore, it is not surprising to observe expression of $P A X 8$ in the proximal portion of the upper urinary tract and in some $(\sim 23 \%)$ of urothelial carcinomas of the renal pelvis. The cells at the basal layer of the ureteral epithelium may represent the less differentiated cells, which still maintain some features of the epithelial cells in the ureteric bud including expression of PAX8 as observed in our study. The decreased PAX8 expression distally along the upper urinary tract might be the result of interaction of the epithelial cells with the surrounding smooth muscle cells, and the consequence of metaplastic changes of the ureteric bud epithelial cells. Although morphologically similar to the epithelium in the renal pelvis and ureters, the urothelium of the urinary bladder and urethra is derived from the ventral urogenital sinus. The urogenital sinus is of endodermal origin ${ }^{37,38}$ and $P A X 8$ has not been detected in this area at any embryonic stages, which is consistent with the lack of PAX8 expression in the urothelium and urothelial carcinomas of the urinary bladder. Whether the urothelial carcinomas of the ureter may express PAX8 needs further investigation in large numbers of tumors from this area.

In addition, $P A X 8$ is expressed in the thyroid diverticulum in the early embryonic stage and is required for the formation and differentiation of the follicular cells. ${ }^{39-41}$ Mutations of $P A X 8$ cause congenital thyroid hypoplasia or absence of thyroid glands ${ }^{42-44} P A X 8$ is detected in the follicular cells of adult human thyroid gland and the follicular cellderived thyroid neoplasm. ${ }^{45-47}$ During embryonic stages, $P A X 8$ is also detected in Müllerian ducts, the embryonic structures adjacent to the Wolffian ducts and the precursor tissues for the female genital tract. In males, the genital tracts derive from the remnant of the Wolffian duct and share a distant common embryonic origin as the kidney. The epithelial cells of female and male genital tracts are positive for $P A X 8$. Mutations of $P A X 8$ cause infertility in male and female mice because of the malformation and obstruction of the genital tracts. ${ }^{48,49}$ PAX8 is also detected in ovarian epithelial carcinomas ${ }^{50}$ and is a useful differential marker from breast carcinomas. ${ }^{51}$ Expression of $P A X 8$ was described in tumors of other tissue origins; ${ }^{29,52,53}$ however, it has not been substantiated by immunohistochemistry in recent publications. ${ }^{29,51} P A X 8$ was not detected in metastatic carcinomas from breast, colon, pancreas, or lung in body fluid (unpublished data). All these evidences support that PAX8 expression is limited to a few normal and neoplastic tissues in a celllineage-dependent manner and may be used as an 
additional immunohistochemical marker for renal epithelial tumors. However, its specificity and sensitivity in diagnostic application await further investigation.

\section{References}

1 Di Loreto C, Di Lauro V, Puglisi F, et al. Immunocytochemical expression of tissue specific transcription factor-1 in lung carcinoma. J Clin Pathol 1997;50: 30-32.

2 Lau SK, Luthringer DJ, Eisen RN. Thyroid transcription factor-1: a review. Appl Immunohistochem Mol Morphol 2002;10:97-102.

3 Barbareschi M, Murer B, Colby TV, et al. CDX-2 homeobox gene expression is a reliable marker of colorectal adenocarcinoma metastases to the lungs. Am J Surg Pathol 2003;27:141-149.

4 Yaziji H, Gown AM. Immunohistochemical markers of melanocytic tumors. Int J Surg Pathol 2003;11:11-15.

5 Kumar S, Perlman E, Harris CA, et al. Myogenin is a specific marker for rhabdomyosarcoma: an immunohistochemical study in paraffin-embedded tissues. Mod Pathol 2000;13:988-993.

6 Cessna MH, Zhou H, Perkins SL, et al. Are myogenin and myoD1 expression specific for rhabdomyosarcoma? A study of 150 cases, with emphasis on spindle cell mimics. Am J Surg Pathol 2001;25:1150-1157.

7 Amin MB, Tamboli P, Javidan J, et al. Prognostic impact of histologic subtyping of adult renal epithelial neoplasms: an experience of 405 cases. Am J Surg Pathol 2002;26:281-291.

8 Skinnider BF, Amin MB. An immunohistochemical approach to the differential diagnosis of renal tumors. Semin Diagn Pathol 2005;22:51-68.

9 Skinnider BF, Folpe AL, Hennigar RA, et al. Distribution of cytokeratins and vimentin in adult renal neoplasms and normal renal tissue: potential utility of a cytokeratin antibody panel in the differential diagnosis of renal tumors. Am J Surg Pathol 2005;29:747-754.

10 Petit A, Castillo M, Santos M, et al. KIT expression in chromophobe renal cell carcinoma: comparative immunohistochemical analysis of KIT expression in different renal cell neoplasms. Am J Surg Pathol 2004;28:676-678.

11 Lin F, Brown RE, Shen T, et al. Immunohistochemical detection of P504S in primary and metastatic renal cell carcinomas. Appl Immunohistochem Mol Morphol 2004;12:153-159.

12 McGregor DK, Khurana KK, Cao C, et al. Diagnosing primary and metastatic renal cell carcinoma: the use of the monoclonal antibody 'Renal Cell Carcinoma Marker'. Am J Surg Pathol 2001;25:1485-1492.

13 Bakshi N, Kunju LP, Giordano T, et al. Expression of renal cell carcinoma antigen (RCC) in renal epithelial and nonrenal tumors: diagnostic implications. Appl Immunohistochem Mol Morphol 2007;15:310-315.

14 Kageyama Y, Sasaki S, Yamamura Y, et al. Water channel protein subtype suggests the origin of renal cell carcinoma. J Urol 1996;156:291-295.

15 Hammerich KH, Ayala GA, Wheeler TM. Application of immunohistochemistry to the genitourinary system (prostate, urinary bladder, testis, and kidney). Arch Pathol Lab Med 2008;132:432-440.
16 George S, Bukowski RM. Biomarkers in clear cell renal cell carcinoma. Expert Rev Anticancer Ther 2007;7: 1737-1747.

17 Al-Ahmadie HA, Alden D, Qin LX, et al. Carbonic anhydrase IX expression in clear cell renal cell carcinoma: an immunohistochemical study comparing 2 antibodies. Am J Surg Pathol 2008;32:377-382.

18 Dressler GR. The cellular basis of kidney development. Annu Rev Cell Dev Biol 2006;22:509-529.

19 Eccles MR, He S, Legge $\mathrm{M}$, et al. PAX genes in development and disease: the role of $P A X 2$ in urogenital tract development. Int J Dev Biol 2002;46: 535-544.

20 Grote D, Souabni A, Busslinger M, et al. Pax 2/8regulated Gata 3 expression is necessary for morphogenesis and guidance of the nephric duct in the developing kidney. Development 2006;133:53-61.

21 Narlis M, Grote D, Gaitan Y, et al. Pax2 and pax8 regulate branching morphogenesis and nephron differentiation in the developing kidney. J Am Soc Nephrol 2007;18:1121-1129.

22 Eccles MR, Wallis LJ, Fidler AE, et al. Expression of the PAX2 gene in human fetal kidney and Wilms' tumor. Cell Growth Differ 1992;3:279-289.

23 Daniel L, Lechevallier E, Giorgi R, et al. Pax-2 expression in adult renal tumors. Hum Pathol 2001;32: 282-287.

24 Mazal PR, Stichenwirth M, Koller A, et al. Expression of aquaporins and PAX-2 compared to CD10 and cytokeratin 7 in renal neoplasms: a tissue microarray study. Mod Pathol 2005;18:535-540.

25 Mazal PR, Schaufler R, Altenhuber-Muller R, et al. Derivation of nephrogenic adenomas from renal tubular cells in kidney-transplant recipients. N Engl J Med 2002;347:653-659.

26 Tong GX, Melamed J, Mansukhani M, et al. PAX2: a reliable marker for nephrogenic adenoma. Mod Pathol 2006;19:356-363.

27 Gokden N, Gokden M, Phan DC, et al. The utility of $P A X-2$ in distinguishing metastatic clear cell renal cell carcinoma from its morphologic mimics: an immunohistochemical study with comparison to renal cell carcinoma marker. Am J Surg Pathol 2008;32: 1462-1467.

28 Poleev A, Fickenscher H, Mundlos S, et al. PAX8, a human paired box gene: isolation and expression in developing thyroid, kidney and Wilms' tumors. Development 1992;116:611-623.

29 Tong GX, Weeden EM, Hamele-Bena D, et al. Expression of $P A X 8$ in nephrogenic adenoma and clear cell adenocarcinoma of the lower urinary tract: evidence of related histogenesis? Am J Surg Pathol 2008;32: 1380-1387.

30 Bouchard M, Souabni A, Mandler M, et al. Nephric lineage specification by Pax 2 and Pax8. Genes Dev 2002;16:2958-2970.

31 Blake JA, Thomas M, Thompson JA, et al. Perplexing pax: from puzzle to paradigm. Dev Dyn 2008;237: 2791-2803.

32 Saxen L, Sariola H. Early organogenesis of the kidney. Pediatr Nephrol 1987;1:385-392.

33 Davies JA, Perera AD, Walker CL. Mechanisms of epithelial development and neoplasia in the metanephric kidney. Int J Dev Biol 1999;43:473-478.

34 Polascik TJ, Bostwick DG, Cairns P. Molecular genetics and histopathologic features of adult distal nephron tumors. Urology 2002;60:941-946. 
35 Droz D, Zachar D, Charbit L, et al. Expression of the human nephron differentiation molecules in renal cell carcinomas. Am J Pathol 1990;137:895-905.

36 Memeo L, Jhang J, Assaad AM, et al. Immunohistochemical analysis for cytokeratin 7, KIT, and PAX2: value in the differential diagnosis of chromophobe cell carcinoma. Am J Clin Pathol 2007;127: 225-229.

37 De La Rosette J, Smedts F, Schoots C, et al. Changing patterns of keratin expression could be associated with functional maturation of the developing human bladder. J Urol 2002;168:709-717.

38 Aboseif S, El-Sakka A, Young P, et al. Mesenchymal reprogramming of adult human epithelial differentiation. Differentiation 1999;65:113-118.

39 Plachov D, Chowdhury K, Walther C, et al. Pax8, a murine paired box gene expressed in the developing excretory system and thyroid gland. Development 1990;110:643-651.

40 Mansouri A, Chowdhury K, Gruss P. Follicular cells of the thyroid gland require $P a x 8$ gene function. Nat Genet 1998;19:87-90.

41 Kang HC, Ohmori M, Harii N, et al. Pax-8 is essential for regulation of the thyroglobulin gene by transforming growth factor-beta1. Endocrinology 2001;142: 267-275.

42 Damante G. Thyroid defects due to Pax8 gene mutations. Eur J Endocrinol 1998;139:563-566.

43 Flamant F, Poguet AL, Plateroti M, et al. Congenital hypothyroid Pax $8(-/-)$ mutant mice can be rescued by inactivating the TRalpha gene. Mol Endocrinol 2002; 16:24-32.

44 Mittag J, Friedrichsen S, Heuer H, et al. Athyroid Pax8-1- mice cannot be rescued by the inactivation of thyroid hormone receptor alpha1. Endocrinology 2005;146:3179-3184.

45 Fabbro D, Di Loreto C, Beltrami CA, et al. Expression of thyroid-specific transcription factors TTF-1 and PAX-8 in human thyroid neoplasms. Cancer Res 1994;54: 4744-4749.

46 Zhang $\mathrm{P}$, Zuo H, Nakamura $\mathrm{Y}$, et al. Immunohistochemical analysis of thyroid-specific transcription factors in thyroid tumors. Pathol Int 2006;56:240-245.

47 Nonaka D, Tang Y, Chiriboga L, et al. Diagnostic utility of thyroid transcription factors $\operatorname{Pax} 8$ and TTF-2 (FoxE1) in thyroid epithelial neoplasms. Mod Pathol 2008;21:192-200.

48 Mittag J, Winterhager E, Bauer K, et al. Congenital hypothyroid female pax8-deficient mice are infertile despite thyroid hormone replacement therapy. Endocrinology 2007;148:719-725.

49 Wistuba J, Mittag J, Luetjens CM, et al. Male congenital hypothyroid Pax8-/- mice are infertile despite adequate treatment with thyroid hormone. J Endocrinol 2007;192:99-109.

50 Bowen NJ, Logani S, Dickerson EB, et al. Emerging roles for $P A X 8$ in ovarian cancer and endosalpingeal development. Gynecol Oncol 2007;104:331-337.

51 Nonaka D, Chiriboga L, Soslow RA. Expression of pax8 as a useful marker in distinguishing ovarian carcinomas from mammary carcinomas. Am J Surg Pathol 2008;32:1566-1571.

52 Pellizzari L, Puppin C, Mariuzzi L, et al. PAX8 expression in human bladder cancer. Oncol Rep 2006; 16:1015-1020.

53 Muratovska A, Zhou C, He S, et al. Paired-box genes are frequently expressed in cancer and often required for cancer cell survival. Oncogene 2003;22:7989-7997. 